\title{
A!
}

This is an electronic reprint of the original article.

This reprint may differ from the original in pagination and typographic detail.

Du, Luojun; Zhang, Qian; Zhang, Tingting; Jia, Zhiyan; Liang, Jing; Liu, Gui Bin; Yang, Rong; Shi, Dongxia; Xiang, Jianyong; Liu, Kaihui; Sun, Zhipei; Yao, Yugui; Zhang, Qingming; Zhang, Guangyu

\section{Robust circular polarization of indirect Q-K transitions in bilayer 3R-W S2}

Published in:

Physical Review B

DOI:

10.1103/PhysRevB.100.161404

Published: 23/10/2019

\section{Document Version}

Publisher's PDF, also known as Version of record

Please cite the original version:

Du, L., Zhang, Q., Zhang, T., Jia, Z., Liang, J., Liu, G. B., Yang, R., Shi, D., Xiang, J., Liu, K., Sun, Z., Yao, Y., Zhang, Q., \& Zhang, G. (2019). Robust circular polarization of indirect Q-K transitions in bilayer 3R-W S2.

Physical Review B, 100(16), [161404]. https://doi.org/10.1103/PhysRevB.100.161404

This material is protected by copyright and other intellectual property rights, and duplication or sale of all or part of any of the repository collections is not permitted, except that material may be duplicated by you for your research use or educational purposes in electronic or print form. You must obtain permission for any other use. Electronic or print copies may not be offered, whether for sale or otherwise to anyone who is not an authorised user. 


\title{
Robust circular polarization of indirect $\mathrm{Q}-\mathrm{K}$ transitions in bilayer $3 R-\mathrm{WS}_{2}$
}

\author{
Luojun Du $\odot,{ }^{1,2}$ Qian Zhang, ${ }^{3}$ Tingting Zhang, ${ }^{1,4}$ Zhiyan Jia, ${ }^{5}$ Jing Liang $\odot,{ }^{6}$ Gui-Bin Liu $\odot,{ }^{4}$ Rong Yang, ${ }^{1}$ Dongxia Shi, ${ }^{1,7}$ \\ Jianyong Xiang, ${ }^{5}$ Kaihui Liu, ${ }^{6}$ Zhipei Sun, ${ }^{2,8}$ Yugui Yao, ${ }^{4}$ Qingming Zhang,,${ }^{1,9,}$ and Guangyu Zhang ${ }^{1,7,10, \dagger}$ \\ ${ }^{1}$ Beijing National Laboratory for Condensed Matter Physics and Institute of Physics, Chinese Academy of Sciences, Beijing 100190, China \\ ${ }^{2}$ Department of Electronics and Nanoengineering, Aalto University, Tietotie 3, FI-02150, Finland \\ ${ }^{3}$ Department of Physics, Renmin University of China, Beijing 100872, China \\ ${ }^{4}$ Beijing Key Laboratory of Nanophotonics and Ultrafine Optoelectronic Systems, School of Physics, Beijing Institute of Technology, \\ Beijing 100081, China \\ ${ }^{5}$ State Key Laboratory for Metastable Materials Science and Technology, Yanshan University, Qinhuangdao 066004, China \\ ${ }^{6}$ State Key Laboratory for Mesoscopic Physics, Collaborative Innovation, Center of Quantum Matter, School of Physics, \\ Peking University, Beijing, China \\ ${ }^{7}$ School of Physical Sciences, University of Chinese Academy of Science, Beijing 100190, China \\ ${ }^{8}$ QTF Centre of Excellence, Department of Applied Physics, Aalto University, FI-00076, Finland \\ ${ }^{9}$ School of Physical Science and Technology, Lanzhou University, Lanzhou 730000, China \\ ${ }^{10}$ Songshan-Lake Materials Laboratory, Dongguan 523808, Guangdong Province, China
}

(Received 28 June 2019; revised manuscript received 9 October 2019; published 23 October 2019)

\begin{abstract}
Valley-contrasting Berry curvature and orbital magnetic moment have led to highly selective circular polarization of direct excitons at the $\mathrm{K}$ valleys in transition-metal dichalcogenides. In addition to $\mathrm{K}$ valleys, $\mathrm{Q}$ valleys, another critical point in the conduction band, also possess well-defined but distinct magnetic moment. Being akin to the direct excitons at $\mathrm{K}$ valleys, indirect excitons associated with $\mathrm{Q}(\mathrm{K})$ valleys in the conduction (valence) band could allow circular polarization in principle. Here, we report an experimental observation of the circular polarization of indirect $\mathrm{Q}-\mathrm{K}$ transitions in noncentrosymmetric bilayer $3 R-\mathrm{WS}_{2}$. In stark contrast to the circular polarization of direct excitons which depolarizes with increasing lattice temperature, the circular polarization of indirect Q-K excitons is extremely robust and independent on the temperature. Such robust circular polarization can be understood as follows: the spin-orbit coupling in the Q valley is much stronger than that in the $\mathrm{K}$ point of the conduction band, significantly suppressing the temperature induced valley depolarization. Our results open up opportunities for exotic valleytronics and quantum information processing applications.
\end{abstract}

DOI: 10.1103/PhysRevB.100.161404

Circular polarization (CP), one of the most fundamental properties in condensed-matter physics, plays a prominent role in generating and detecting a cornucopia of phenomena, such as spin-Hall effect, valley-Hall effect, exciton-Hall effect, and chiral light-emitting transistors [1-8]. It is highly desired to quest for a system manifesting robust CP. Due to the combination of time-reversal symmetry and explicitly broken spatial-inversion symmetry, $\mathrm{K}$ and $\mathrm{K}^{\prime}$ valleys of monolayer transition-metal dichalcogenides (TMDCs) enable contrasting Berry curvature and magnetic moment, giving rise to valleyselective $\mathrm{CP}$ of direct excitons at the corners of a hexagonal Brillouin zone [9-15]. Besides $\mathrm{K}$ valleys, six nonequivalent $\mathrm{Q}$ pockets related by both threefold rotational symmetry and time-reversal symmetry are also the critical points of the conduction band (CB) [16-18]. These six $Q$ valleys form two groups $\left(\mathrm{Q}\right.$ and $\left.\mathrm{Q}^{\prime}\right)$ and also possess contrasting spin, Berry curvature, and magnetic moment [19-21], demonstrated recently by valley-dependent circularly polarized light selection

\footnotetext{
*Corresponding author: qmzhang@ ruc.edu.cn

†'gyzhang@iphy.ac.cn
}

rule, valley Zeeman effect, and circular photogalvanic effect [21-23]. Therefore, indirect $\mathrm{Q}-\mathrm{K}\left(\mathrm{Q}^{\prime}-\mathrm{K}^{\prime}\right)$ transitions associated with $\mathrm{Q}\left(\mathrm{Q}^{\prime}\right)$ in the $\mathrm{CB}$ and $\mathrm{K}\left(\mathrm{K}^{\prime}\right)$ valleys in the valence band (VB) could possess $\mathrm{CP}$ in principle.

However, due to the direct-gap feature of monolayer at $\mathrm{K}$ points [24,25], CP of indirect Q-K transitions cannot be achieved. In marked contrast to $\mathrm{K}$ valleys that are predominantly from the $d$ orbital of transition metals, Q valleys possess considerable $p_{z}$ character of chalcogens, which would lower the energy and cause $\mathrm{Q}$ valleys to be the conductionband minimum (CBM) for bilayer and thick layer [16-18]. Although $\Gamma$ point in the VB also harbors the $p_{z}$ character of chalcogens that leads to the energy upshift, the strong spinorbit coupling in tungsten-based TMDCs makes the valenceband maximum (VBM) of a bilayer still at K points [17,26]. Therefore, tungsten-based bilayer TMDCs provide a perfect platform for indirect Q-K transitions [17,27-29]. However, in usual $2 \mathrm{H}$ stacked bilayer TMDCs, orbital magnetic moment vanishes due to the restoration of spatial-inversion symmetry [30,31]. Moreover, considerable $p_{z}$ character of chalcogens at $Q$ valleys leads to significant interlayer hopping and seriously weakens the hidden spin polarization which gives rise to the 

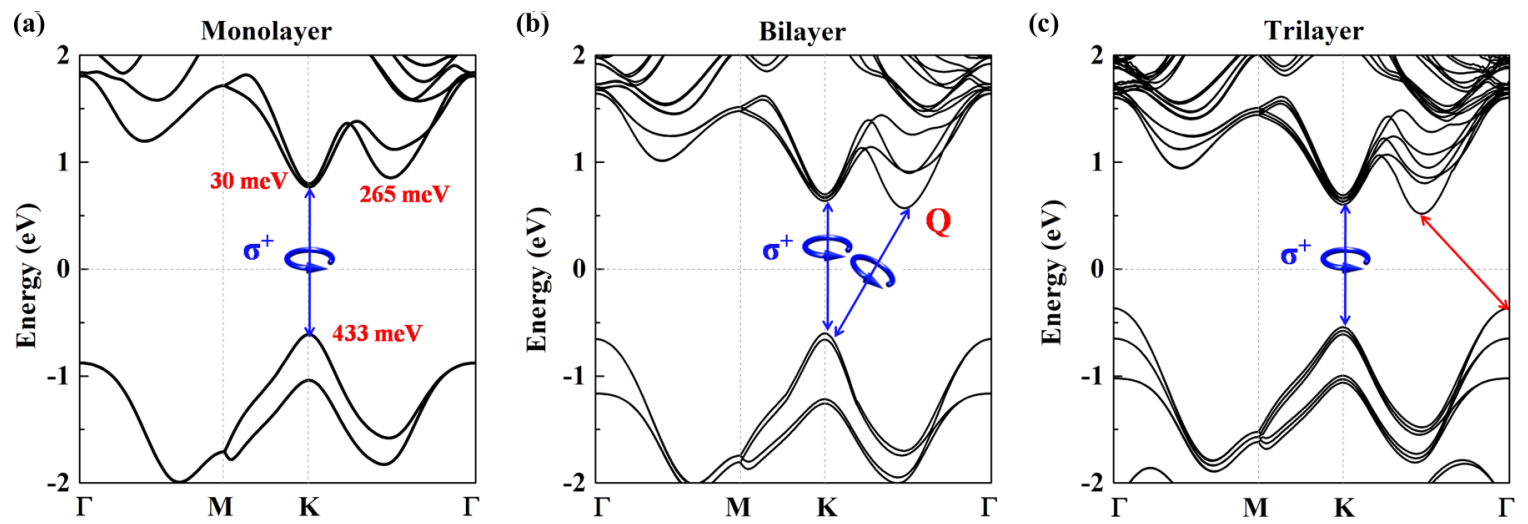

FIG. 1. Layer-dependent electronic structures of $3 R$ stacking $\mathrm{WS}_{2}$. (a) Band structure of monolayer WS $\mathrm{W}_{2}$. Both CBM and VBM are located at the $\mathrm{K}$ point. The spin-orbit splitting of $\mathrm{CB}, \mathrm{VB}$, and $\mathrm{Q}$ point are overlaid. (b) Electronic band of bilayer $3 R-\mathrm{WS} 2 . \mathrm{CBM}$ and $\mathrm{CBM}$ locate at the $\mathrm{Q}$ and $\mathrm{K}$ points, respectively. (c) Electronic structure of trilayer $3 R$-WS $\mathrm{W}_{2}$ CBM and VBM locate at the Q and $\Gamma$ points, respectively. Fermi level is set to zero.

CP of direct excitons [17,30-38]. Thus, the $\mathrm{CP}$ of the indirect $\mathrm{Q}-\mathrm{K}$ transitions in $2 \mathrm{H}$ stacked bilayer TMDCs cannot be acquired [33-35].

In addition to $2 \mathrm{H}$ stacking, TMDCs have another crystal structure, i.e., $3 R$ stacking [37,39-41]. Compared with $2 \mathrm{H}$ stacking, the critical points are the same in $3 R$ stacking since the strengths of interlayer interaction between the two stacking structures are almost equal to each other $[29,42]$. Strikingly, the inversion symmetry is explicitly broken in $3 R$ stacked bilayer TMDCs $[40,41]$, giving rise to valleycontrasting Berry curvature and orbital magnetic moment $[10,19,43]$. As a consequence, tungsten-based bilayer TMDCs with $3 R$ stacking phase offer an unprecedented platform to demonstrate the $\mathrm{CP}$ of indirect $\mathrm{Q}-\mathrm{K}$ transitions. Here, we demonstrate the $\mathrm{CP}$ of indirect $\mathrm{Q}-\mathrm{K}$ transitions in $3 R$ stacked bilayer $\mathrm{WS}_{2}$. In striking contrast to the monotonic decrease of $\mathrm{CP}$ for direct excitons or the absence of $\mathrm{CP}$ for indirect $\mathrm{Q}-\Gamma$ transitions, the indirect $\mathrm{Q}-\mathrm{K}$ transitions harbor robust temperature-independent $\mathrm{CP}$, which would be fundamentally important in searching for valleytronics and chiral lightemitting transistor applications.

We first performed density-functional theory (DFT) calculations (see details in the Supplemental Material [44]) to investigate the thickness-driven evolution of electronic structures and the essential difference between $3 R$ and $2 \mathrm{H}$ stacking. Figure 1 presents the results of monolayer, bilayer, and trilayer $3 R-\mathrm{WS}_{2}$. In good agreement with previous works $[45,46]$, our results show that monolayer $\mathrm{WS}_{2}$ possesses a direct gap at $\mathrm{K}$ valleys coupled with circularly polarized light [Fig. 1(a)]. Moreover, it can be known that the spin-orbit splitting is 433, 30 , and $265 \mathrm{meV}$ for the $\mathrm{VB}$ at the $\mathrm{K}$ point, and $\mathrm{CB}$ at the $\mathrm{K}$ and $\mathrm{Q}$ points, respectively. Compared with the $\mathrm{CB}$ at the $\mathrm{K}$ points, the much stronger spin-orbit splitting at the $\mathrm{Q}$ points would play a key role in $\mathrm{CP}$, as will be seen below. By increasing the thickness from monolayer to bilayer or trilayer $3 R$-WS $\mathrm{WS}_{2}$, we can see the crossover from direct to indirect [Figs. 1(b) and 1(c)]. The CBM changes from $\mathrm{K}$ point in monolayer to $\mathrm{Q}$ valley in bilayer/trilayer $3 R-\mathrm{WS}_{2}$. Strikingly, a quite distinct VBM between bilayer and trilayer $3 R$-WS $\mathrm{W}_{2}$ can be observed. The VBM is still at $\mathrm{K}$ point for bilayer [Fig. 1(b)] while becomes the $\Gamma$ point in trilayer
[Fig. 1(c)]. According to these results, the indirect excitons are Q-K and Q- $\Gamma$ transitions for bilayer and trilayer $3 R-\mathrm{WS}_{2}$, as illustrated in Figs. 1(b) and 1(c), respectively. Such distinct indirect excitons between bilayer and trilayer $3 R$ - $\mathrm{WS}_{2}$ are further confirmed via temperature-driven evolution of photoluminescence (PL), since distinct critical points harbor different temperature responses (see Supplemental Material for more details [44]). In addition, the Berry curvature of the $\Gamma$ point is null, thus the indirect $Q-\Gamma$ transitions in trilayer would show no $\mathrm{CP}$, which can be viewed as a perfect reference.

Since bulk $\mathrm{WS}_{2}$ crystal is usually $2 \mathrm{H}$ phase, it is difficult to obtain $3 R$ stacked bilayer $\mathrm{WS}_{2}$ through mechanical exfoliation. Fortunately, the chemical vapor deposition (CVD) method provides an efficient path to obtain $3 R$ stacked TMDCs $[42,47]$. Here, $\mathrm{WS}_{2}$ layers with $3 R$ stacking are obtained by the CVD process on $90-\mathrm{nm} \mathrm{SiO}_{2} / \mathrm{Si}$ substrates [48], using sulfur and tungsten trioxide powder as precursors (see Supplemental Material for more details [44]). Figure 2(a) shows the optical micrograph of representative samples with the thickness overlaid. The number of layers is first visually identified by observing their interference color through the optical microscope and further confirmed by PL and Raman spectra. The evolution of PL versus the number of layers is presented in Fig. 2(b). The PL spectrum of monolayer consists of a single narrow feature centered at $1.964 \mathrm{eV}$ with the strongest quantum efficiency, indicating the direct-gap feature $[46,48,49]$. In marked contrast, PL spectra from bilayer and trilayer flakes display not only direct-gap transitions (higher energy), but also indirect excitons (lower energy). The indirect band gap locates at $1.62 \mathrm{eV}(1.54 \mathrm{eV})$ for bilayer (trilayer), in fair agreement with previous results [46,48]. Figure 2(c) presents the Raman spectra excited by $1.96 \mathrm{eV}$ radiation on resonance with the $A$ exciton, which provides a reliable fingerprint to determine the thickness since the out-of-plane $A_{1 g}(\Gamma)$ mode of $\mathrm{WS}_{2}$ splits into $N$ components for $N$ layers $[48,50]$. It can be seen clearly, from the Lorentzian fitting (solid lines), that $A_{1 g}(\Gamma)$ mode spectra have one, two, and three components for monolayer, bilayer, and trilayer $3 R-\mathrm{WS}_{2}$, respectively. Strikingly, from the optical micrograph [Fig. 2(a)], it can be known that the crystal orientations of distinct layers in bilayer and trilayer are the same. Thus, we believe that our $\mathrm{WS}_{2}$ 
(a)

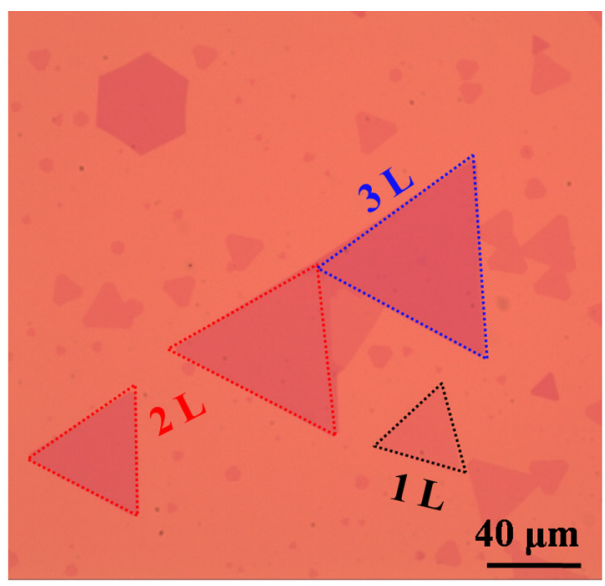

(c)

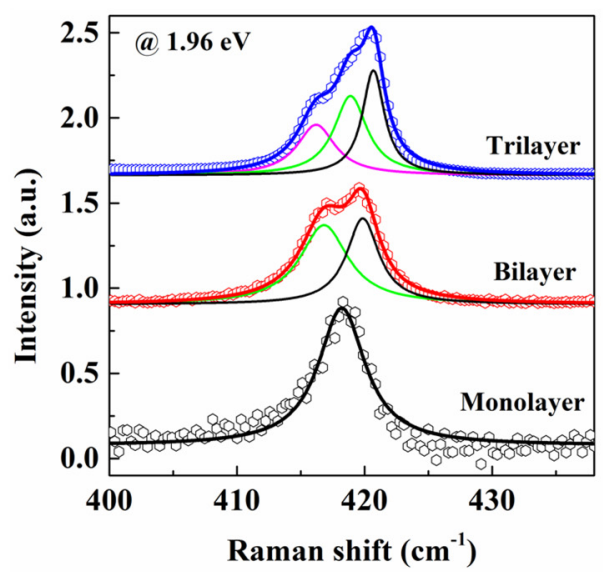

(b)

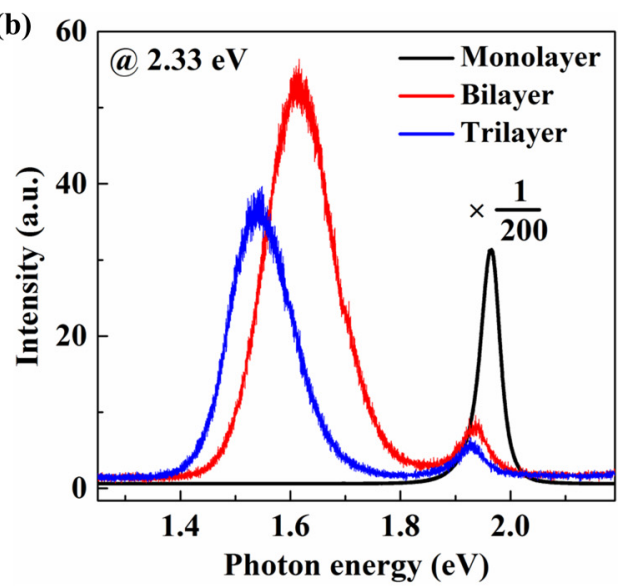

(d)

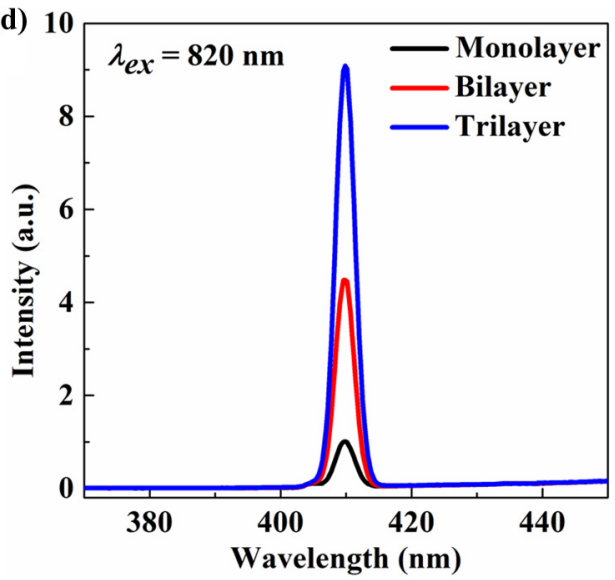

FIG. 2. Microscopy and characterization of monolayer, bilayer, and trilayer $3 R$-WS $\mathrm{W}_{2}$. (a) Optical micrograph of the representative $3 R$-WS samples. Monolayer, bilayer, and trilayer samples are highlighted by black, red, and blue dashed lines, respectively. Scale bar is $40 \mu \mathrm{m}$. (b) PL spectra of monolayer, bilayer, and trilayer $3 R-\mathrm{WS}_{2}$ at $2.33 \mathrm{eV}$ excitation. (c) Raman spectra of monolayer, bilayer, and trilayer $3 R$-WS under $1.96 \mathrm{eV}$ excitation, on resonance with the $A$ exciton. Lorentzian fitting of the $A_{1 g}(\Gamma)$ phonon modes are shown. (d) Layer-dependent SHG spectra of $3 R-\mathrm{WS}_{2}$ under the excitation wavelength $\left(\lambda_{\mathrm{ex}}\right)$ of $820 \mathrm{~nm}$.

samples should be $3 R$ phase. In order to verify this, we performed second-harmonic generation (SHG) measurement which is a sensitive probe to the crystalline symmetry [40,41,51]. Figure 2(d) presents the layer-dependent SHG spectra of our $\mathrm{WS}_{2}$ samples under an excitation wavelength of $\lambda_{e x}=820 \mathrm{~nm}$. The SHG intensity of bilayer (trilayer) is four (nine) times stronger than that of monolayer, unequivocally confirming that our $\mathrm{WS}_{2}$ samples are $3 R$ stacking [40,41,47,52].

Having obtained the bilayer $3 R-\mathrm{WS}_{2}$, we then focus on the $\mathrm{CP}$ of indirect $\mathrm{Q}-\mathrm{K}$ transitions associated with $\mathrm{Q}(\mathrm{K})$ valleys in $\mathrm{CB}(\mathrm{VB})$. Figure 3 presents the polarization-resolved normalized PL spectra (left circularly polarized $\sigma^{+}$and right circularly polarized $\sigma^{-}$) of bilayer [Fig. 3(a)] and trilayer [Fig. 3(b)] $3 R-\mathrm{WS}_{2}$ at 10 and $300 \mathrm{~K}$, excited by $\sigma^{+}$radiation with energy of $2.33 \mathrm{eV}$. We quantify the magnitude of $\mathrm{CP}$, i.e., the anisotropy of circularly polarized PL, as follows $[11,12]$ :

$$
\rho=\frac{\mathrm{I}\left(\sigma^{+}\right)-\mathrm{I}\left(\sigma^{-}\right)}{\mathrm{I}\left(\sigma^{+}\right)+\mathrm{I}\left(\sigma^{-}\right)}
$$

where $I\left(\sigma^{ \pm}\right)$is the intensity of the left- (right-) handed circular-polarization component. Being good congruent with previous analysis, the indirect $\mathrm{Q}-\Gamma$ transitions of trilayer
$3 R-\mathrm{WS}_{2}$ are unpolarized at either 10 or $300 \mathrm{~K}$ [Fig. 3(b)]. In marked contrast, the indirect $\mathrm{Q}-\mathrm{K}$ transitions in bilayer $3 R-\mathrm{WS}_{2}$, in close analogy to the direct $A$ excitons of bilayer and trilayer, hold highly selective $\mathrm{CP}$ at both 10 and $300 \mathrm{~K}$. Our results demonstrate the $\mathrm{CP}$ of indirect $\mathrm{Q}-\mathrm{K}$ transitions, which would play a prominent role for exotic optoelectronic applications. More importantly, from the dashed horizontal lines illustrated in Fig. 3(a), it is evident that the normalized intensity of the $\sigma^{-}$component for indirect Q-K transitions is larger (smaller) than that for direct $A$ excitons in bilayer $3 R-\mathrm{WS}_{2}$ at $10 \mathrm{~K}(300 \mathrm{~K})$. This indicates that the $\mathrm{CP}$ of indirect $\mathrm{Q}-\mathrm{K}$ transitions is weaker than that of direct $A$ excitons in $10 \mathrm{~K}$, but stronger than $A$ excitons at $300 \mathrm{~K}$, demonstrating more robust $\mathrm{CP}$ for indirect $\mathrm{Q}-\mathrm{K}$ transitions at room temperature.

Figure 4 presents the temperature-driven evolution of $\mathrm{CP}$ for the direct $A$ and indirect excitons of bilayer and trilayer $3 R$-WS $\mathrm{W}_{2}$. Within experimental measurement error, the CP of indirect $\mathrm{Q}-\Gamma$ transitions of trilayer $3 R-\mathrm{WS}_{2}$ is null at all temperatures. In contrast, we can observe highly selective $\mathrm{CP}$ for direct $A$ excitons of both bilayer and trilayer, and indirect $\mathrm{Q}-\mathrm{K}$ transitions in bilayer $3 R-\mathrm{WS}_{2}$. Interestingly, a distinct temperature response between the direct $A$ excitons and in- 

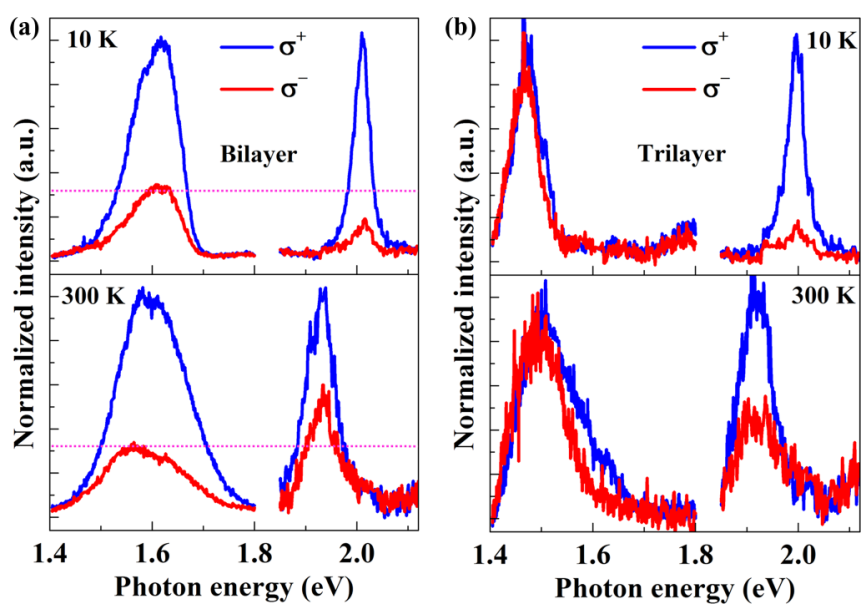

FIG. 3. Robust CP of indirect Q-K transitions. $\sigma^{+}$(blue) and $\sigma^{-}$ (red) resolved PL spectra for bilayer $3 R-\mathrm{WS}_{2}$ (a) and trilayer $3 R-\mathrm{WS}_{2}$ (b) at $10 \mathrm{~K}$ (upper panel) and $300 \mathrm{~K}$ (lower panel). The intensities more than (less than) $1.82 \mathrm{eV}$ are normalized by the intensity of the direct (indirect) exciton with $\sigma^{+}$component.

direct Q-K transitions is observed. For the $A$ excitons of both bilayer and trilayer $3 R-\mathrm{WS}_{2}$, the $\mathrm{CP}$ decreases monotonously with increasing temperature, being consistent with the results of $3 R-\mathrm{MoS}_{2}$ [37]. In marked contrast, the $\mathrm{CP}$ of indirect Q-K transitions in bilayer $3 R-\mathrm{WS}_{2}$ is independent on the temperature. Such robust CP of indirect Q-K transitions could broaden the $\mathrm{CP}$ sources and play a key role for valleytronics $[1,7]$.

Under steady-state circularly polarized light excitation, the magnitude of CP is determined by the exciton lifetime $\left(\tau_{E}\right)$ and valley lifetime $\left(\tau_{V}\right)$ and can be approximated as $\rho=$ $\frac{\rho_{0}}{1+2 \tau_{E} / \tau_{V^{\prime}}}$, where $\rho_{0}$ denotes the initially generated polarization

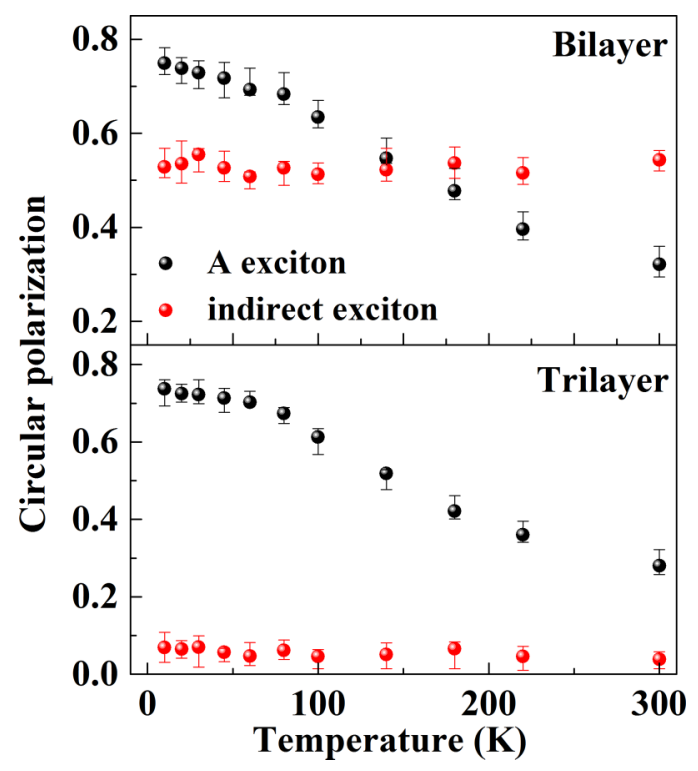

FIG. 4. The $\mathrm{CP}$ of the direct $A$ exciton (black) and the indirect exciton (red) as a function of temperature for bilayer (upper panel) and trilayer (lower panel) $3 R$-WS 2 . The error bar stems from multiple measurements of at least three samples.
$[12,15,33]$. Due to the conservation of lattice momentum for optical transitions, $\tau_{E}$ of the direct excitons is much shorter than that of the indirect excitons. Recent time-resolved PL spectra revealed that $\tau_{E}$ of the indirect transition in bilayer TMDCs is an order of magnitude longer than that of direct transitions [35]. Therefore, the direct excitons, compared with indirect $\mathrm{Q}-\mathrm{K}$ transitions, can possess a higher magnitude of $\mathrm{CP}$ at low temperature. When elevating the temperature, it has intrinsic impacts on both the $\tau_{E}$ and $\tau_{V}$, giving rise to the temperature-dependent CP. First, as the spin-orbit splitting of $\mathrm{CB}$ is only $30 \mathrm{meV}$ for the $\mathrm{K}$ valley, the spin at finite momentum is not a perfect quantum number and can flip with a quick process. The spin flip of electrons could lead to spin flip of holes via strong electron-hole Coulomb exchange interaction accompanying intervalley scattering, which annihilates an exciton in one valley and creates another exciton in the other valley [53-56]. The valley depolarization at $\mathrm{K}$ points typically increases with raising the temperature, leading to the decrease of $\tau_{V}$ and CP [54,55]. In contrast, the spin-orbit splitting of the $\mathrm{Q}$ valley $(265 \mathrm{meV})$ is an order of magnitude larger than that of the $\mathrm{K}$ valley in $\mathrm{CB}$. As a consequence, the spin at the $\mathrm{Q}$ valley can be viewed as a good quantum number and spin flip of electrons is strongly suppressed. On the other hand, temperature can strongly affect the $\tau_{E}$. For direct excitons, the $\tau_{E}$ increases with the temperature [57], causing the decrease of CP at elevated temperatures. However, the $\tau_{E}$ of indirect Q-K transitions is independent on the temperature [35], which can be confirmed by the temperature-independent intensity of indirect excitons (see Supplemental Material for more details [44]). Both the temperature-independent $\tau_{E}$ and $\tau_{V}$ lead to the observation of temperature robust $\mathrm{CP}$ for indirect $\mathrm{Q}-\mathrm{K}$ transitions.

In addition, the indirect Q-K transitions, in principle, could possess valley Zeeman effect due to the contrasting Berry curvature and magnetic moment between $\mathrm{Q}$ and $\mathrm{Q}^{\prime}$ valleys [58-62]. Moreover, the indirect $\mathrm{Q}-\mathrm{K}$ transitions provide a promising playground for observing the intercellular contribution arising from the phase winding of the Bloch wave packet (see Supplemental Material for more details [44]), in stark contrast to the direct $A$ excitons in that the valley Zeeman effect stems predominantly from the intracellular contribution [58-63]. Unfortunately, the quantitative Zeeman shift of indirect Q-K transitions is masked by the asymmetry and large full width at half maximum (see Supplemental Material for more details [44]). Further experiments with high magnetic field would help us to elaborate clearly the Zeeman effects of indirect Q-K transitions [64-66].

To summarize, our results uncover the $\mathrm{CP}$ of indirect $\mathrm{Q}-\mathrm{K}$ transitions in noncentrosymmetric bilayer $3 R-\mathrm{WS}_{2}$. In contrast to the fact that the $\mathrm{CP}$ of direct $A$ excitons decreases monotonously with increasing temperature, the indirect Q-K transitions harbor robust and temperature-independent $\mathrm{CP}$. Such robust CP paves a way for prospects in optical control of exotic spintronics and valleytronics applications at room temperature.

G.Z. acknowledges the financial support from NSF of China (Grants No. 11834017 and No. 61888102), the Strategic Priority Research Program of CAS (XDB30000000), 
the Key Research Program of Frontier Sciences of CAS (QYZDB-SSW-SLH004), and the National Key R\&D program (2016YFA0300904). Q.Z. acknowledges support from the Ministry of Science and Technology of China (2017YFA0302904 and 2016YFA0300504) and the NSF of China (11774419). Z.S. acknowledges the financial support from Business Finland (A-Photonics), Academy of Finland (Grants No. 276376, No. 284548, No. 286920, No. 295777, No. 298297, No. 304666, No. 312297, No. 312551, and No. 314810), Academy of Finland Flagship Programme (320167, PREIN), the European Union's Horizon 2020 research and innovation programme (820423, S2QUIP).
[1] J. Sinova, S. O. Valenzuela, J. Wunderlich, C. Back, and T. Jungwirth, Rev. Mod. Phys. 87, 1213 (2015).

[2] J. Wunderlich, B.-G. Park, A. C. Irvine, L. P. Zârbo, E. Rozkotová, P. Nemec, V. Novák, J. Sinova, and T. Jungwirth, Science 330, 1801 (2010).

[3] K. F. Mak, K. L. McGill, J. Park, and P. L. McEuen, Science 344, 1489 (2014).

[4] M. Onga, Y. Zhang, T. Ideue, and Y. Iwasa, Nat. Mater. 16, 1193 (2017).

[5] T. D. Nguyen, E. Ehrenfreund, and Z. V. Vardeny, Science 337, 204 (2012).

[6] Y. Zhang, T. Oka, R. Suzuki, J. Ye, and Y. Iwasa, Science 344, 725 (2014).

[7] K. F. Mak, D. Xiao, and J. Shan, Nat. Photonics 12, 451 (2018).

[8] J. R. Schaibley, H. Yu, G. Clark, P. Rivera, J. S. Ross, K. L. Seyler, W. Yao, and X. Xu, Nat. Rev. Mater. 1, 16055 (2016).

[9] W. Yao, D. Xiao, and Q. Niu, Phys. Rev. B 77, 235406 (2008).

[10] D. Xiao, G.-B. Liu, W. Feng, X. Xu, and W. Yao, Phys. Rev. Lett. 108, 196802 (2012).

[11] H. Zeng, J. Dai, W. Yao, D. Xiao, and X. Cui, Nat. Nanotechnol. 7, 490 (2012).

[12] K. F. Mak, K. He, J. Shan, and T. F. Heinz, Nat. Nanotechnol. 7, 494 (2012).

[13] G. Sallen, L. Bouet, X. Marie, G. Wang, C. R. Zhu, W. P. Han, Y. Lu, P. H. Tan, T. Amand, B. L. Liu, and B. Urbaszek, Phys. Rev. B 86, 081301(R) (2012).

[14] A. M. Jones, H. Yu, N. J. Ghimire, S. Wu, G. Aivazian, J. S. Ross, B. Zhao, J. Yan, D. G. Mandrus, D. Xiao, W. Yao, and X. Xu, Nat. Nanotechnol. 8, 634 (2013).

[15] L. Du, Q. Zhang, B. Gong, M. Liao, J. Zhu, H. Yu, R. He, K. Liu, R. Yang, D. Shi, L. Gu, F. Yan, G. Zhang, and Q. Zhang, Phys. Rev. B 97, 115445 (2018).

[16] G.-B. Liu, D. Xiao, Y. Yao, X. Xu, and W. Yao, Chem. Soc. Rev. 44, 2643 (2015).

[17] Z. Gong, G.-B. Liu, H. Yu, D. Xiao, X. Cui, X. Xu, and W. Yao, Nat. Commun. 4, 2053 (2013).

[18] L. Du, T. Zhang, M. Liao, G. Liu, S. Wang, R. He, Z. Ye, H. Yu, R. Yang, D. Shi, Y. Yao, and G. Zhang, Phys. Rev. B 97, 165410 (2018).

[19] W. Feng, Y. Yao, W. Zhu, J. Zhou, W. Yao, and D. Xiao, Phys. Rev. B 86, 165108 (2012).

[20] H. Liu, J. Chen, H. Yu, F. Yang, L. Jiao, G.-B. Liu, W. Ho, C. Gao, J. Jia, W. Yao, and M. Xie, Nat. Commun. 6, 8180 (2015).

[21] Z. Wu, S. Xu, H. Lu, A. Khamoshi, G.-B. Liu, T. Han, Y. Wu, J. Lin, G. Long, Y. He, Y. Cai, Y. Yao, F. Zhang, and N. Wang, Nat. Commun. 7, 12955 (2016).

[22] H. Yuan, X. Wang, B. Lian, H. Zhang, X. Fang, B. Shen, G. Xu, Y. Xu, S.-C. Zhang, H. Y. Hwang, and Y. Cui, Nat. Nanotechnol. 9, 851 (2014).

[23] Y. Tatsumi and R. Saito, Phys. Rev. B 97, 115407 (2018).
[24] K. F. Mak, C. Lee, J. Hone, J. Shan, and T. F. Heinz, Phys. Rev. Lett. 105, 136805 (2010).

[25] A. Splendiani, L. Sun, Y. Zhang, T. Li, J. Kim, C.-Y. Chim, G. Galli, and F. Wang, Nano Lett. 10, 1271 (2010).

[26] Y. Zhang, M. M. Ugeda, C. Jin, S.-F. Shi, A. J. Bradley, A. Martín-Recio, H. Ryu, J. Kim, S. Tang, Y. Kim, B. Zhou, C. Hwang, Y. Chen, F. Wang, M. F. Crommie, Z. Hussain, Z.-X. Shen, and S.-K. Mo, Nano Lett. 16, 2485 (2016).

[27] Z. Wang, Y.-H. Chiu, K. Honz, K. F. Mak, and J. Shan, Nano Lett. 18, 137 (2018).

[28] J. Lindlau, M. Selig, A. Neumann, L. Colombier, J. Förste, V. Funk, M. Förg, J. Kim, G. Berghäuser, T. Taniguchi, K. Watanabe, F. Wang, E. Malic, and A. Högele, Nat. Commun. 9, 2586 (2018).

[29] L. M. Schneider, J. Kuhnert, S. Schmitt, W. Heimbrodt, U. Huttner, L. Meckbach, T. Stroucken, S. W. Koch, S. Fu, X. Wang, K. Kang, E.-H. Yang, and A. Rahimi-Iman, arXiv:1905.02814.

[30] J. Lee, K. F. Mak, and J. Shan, Nat. Nanotechnol. 11, 421 (2016).

[31] L. Du, M. Liao, G.-B. Liu, Q. Wang, R. Yang, D. Shi, Y. Yao, and G. Zhang, Phys. Rev. B 99, 195415 (2019).

[32] Q. Liu, X. Zhang, and A. Zunger, Phys. Rev. Lett. 114, 087402 (2015).

[33] B. Zhu, H. Zeng, J. Dai, Z. Gong, and X. Cui, Proc. Natl. Acad. Sci. U.S.A. 111, 11606 (2014).

[34] A. M. Jones, H. Yu, J. S. Ross, P. Klement, N. J. Ghimire, J. Yan, D. G. Mandrus, W. Yao, and X. Xu, Nat. Phys. 10, 130 (2014).

[35] G. Wang, X. Marie, L. Bouet, M. Vidal, A. Balocchi, T. Amand, D. Lagarde, and B. Urbaszek, Appl. Phys. Lett. 105, 182105 (2014).

[36] S. Wu, J. S. Ross, G.-B. Liu, G. Aivazian, A. Jones, Z. Fei, W. Zhu, D. Xiao, W. Yao, D. Cobden, and X. Xu, Nat. Phys. 9, 149 (2013).

[37] R. Suzuki, M. Sakano, Y. J. Zhang, R. Akashi, D. Morikawa, A. Harasawa, K. Yaji, K. Kuroda, K. Miyamoto, T. Okuda, K. Ishizaka, R. Arita, and Y. Iwasa, Nat. Nanotechnol. 9, 611 (2014).

[38] X. Zhang, Q. Liu, J.-W. Luo, A. J. Freeman, and A. Zunger, Nat. Phys. 10, 387 (2014).

[39] Q. H. Wang, K. Kalantar-Zadeh, A. Kis, J. N. Coleman, and M. S. Strano, Nat. Nanotechnol. 7, 699 (2012).

[40] M. Zhao, Z. Ye, R. Suzuki, Y. Ye, H. Zhu, J. Xiao, Y. Wang, Y. Iwasa, and X. Zhang, Light Sci. Appl. 5, e16131 (2016).

[41] J. Shi, P. Yu, F. Liu, P. He, R. Wang, L. Qin, J. Zhou, X. Li, J. Zhou, X. Sui, S. Zhang, Y. Zhang, Q. Zhang, T. C. Sum, X. Qiu, Z. Liu, and Xinfeng Liu, Adv. Mater. 29, 1701486 (2017).

[42] K. Liu, L. Zhang, T. Cao, C. Jin, D. Qiu, Q. Zhou, A. Zettl, P. Yang, S. G. Louie, and Feng Wang, Nat. Commun. 5, 4966 (2014). 
[43] D. Xiao, W. Yao, and Q. Niu, Phys. Rev. Lett. 99, 236809 (2007).

[44] See Supplemental Material at http://link.aps.org/supplemental/ 10.1103/PhysRevB.100.161404, which includes Refs. $[9,10,16,18,30,33,36,37,41,48,50,58-75]$, for details on DFT calculations, the essential difference between the electronic structure of $3 R$ and $2 \mathrm{H}$ stacking concerning chemical vapor deposition, Raman measurement, the evolution of photon energy with temperature, valley Zeeman splitting, and photon energy dependent $\mathrm{CP}$.

[45] A. Kormányos, G. Burkard, M. Gmitra, J. Fabian, V. Zólyomi, N. D. Drummond, and V. Fal'ko, 2D Mater. 2, 022001 (2015).

[46] H. Zeng, G.-B. Liu, J. Dai, Y. Yan, B. Zhu, R. He, L. Xie, S. Xu, X. Chen, W. Yao, and X. Cui, Sci. Rep. 3, 1608 (2013).

[47] D. Yang, X. Hu, M. Zhuang, Y. Ding, S. Zhou, A. Li, Y. Yu, H. Li, Z. Luo, L. Gan, and T. Zhai, Adv. Funct. Mater. 28, 1800785 (2018).

[48] L. Du, Z. Jia, Q. Zhang, A. Zhang, T. Zhang, R. He, R. Yang, D. Shi, Y. Yao, J. Xiang, G. Zhang, and Q. Zhang, 2D Mater. 5, 035028 (2018).

[49] L. Du, Y. Zhao, Z. Jia, M. Liao, Q. Wang, X. Guo, Z. Shi, R. Yang, K. Watanabe, T. Taniguchi, J. Xiang, D. Shi, Q. Dai, Z. Sun, and G. Zhang, Phys. Rev. B 99, 205410 (2019).

[50] M. Staiger, R. Gillen, N. Scheuschner, O. Ochedowski, F. Kampmann, M. Schleberger, C. Thomsen, and J. Maultzsch, Phys. Rev. B 91, 195419 (2015).

[51] Y. Li, Y. Rao, K. F. Mak, Y. You, S. Wang, C. R. Dean, and T. F. Heinz, Nano Lett. 13, 3329 (2013).

[52] A. Autere, H. Jussila, Y. Dai, Y. Wang, H. Lipsanen, and Z. Sun, Adv. Mater. 30, 1705963 (2018).

[53] K. Hao, G. Moody, F. Wu, C. K. Dass, L. Xu, C.-H. Chen, L. Sun, M.-Y. Li, L.-J. Li, A. H. MacDonald, and X. Li, Nat. Phys. 12, 677 (2016).

[54] C. R. Zhu, K. Zhang, M. Glazov, B. Urbaszek, T. Amand, Z. W. Ji, B. L. Liu, and X. Marie, Phys. Rev. B 90, 161302(R) (2014).

[55] J. Kim, C. Jin, B. Chen, H. Cai, T. Zhao, P. Lee, S. Kahn, K. Watanabe, T. Taniguchi, S. Tongay, M. F. Crommie, and F. Wang, Sci. Adv. 3, e1700518 (2017).

[56] C. Jin, E. Y. Ma, O. Karni, E. C. Regan, F. Wang, and T. F. Heinz, Nat. Nanotechnol. 13, 994 (2018).

[57] T. Korn, S. Heydrich, M. Hirmer, J. Schmutzler, and C. Schüller, Appl. Phys. Lett. 99, 102109 (2011).

[58] G. Aivazian, Z. Gong, A. M. Jones, R.-L. Chu, J. Yan, D. G. Mandrus, C. Zhang, D. Cobden, W. Yao, and X. Xu, Nat. Phys. 11, 148 (2015).
[59] A. Srivastava, M. Sidler, A. V. Allain, D. S. Lembke, A. Kis, and A. Imamoğlu, Nat. Phys. 11, 141 (2015).

[60] D. MacNeill, C. Heikes, K. F. Mak, Z. Anderson, A. Kormányos, V. Zólyomi, J. Park, and D. C. Ralph, Phys. Rev. Lett. 114, 037401 (2015).

[61] Y. Li, J. Ludwig, T. Low, A. Chernikov, X. Cui, G. Arefe, Y. D. Kim, A. M. van der Zande, A. Rigosi, H. M. Hill, S. H. Kim, J. Hone, Z. Li, D. Smirnov, and T. F. Heinz, Phys. Rev. Lett. 113, 266804 (2014).

[62] M. Koperski, M. R. Molas, A. Arora, K. Nogajewski, A. O. Slobodeniuk, C. Faugeras, and M. Potemski, Nanophotonics 6, 1289 (2017).

[63] P. Nagler, M. V. Ballottin, A. A. Mitioglu, M. V. Durnev, T. Taniguchi, K. Watanabe, A. Chernikov, C. Schüller, M. M. Glazov, P. C. M. Christianen, and T. Korn, Phys. Rev. Lett. 121, 057402 (2018).

[64] G. Plechinger, P. Nagler, A. Arora, A. G. del Águila, M. V. Ballottin, T. Frank, P. Steinleitner, M. Gmitra, J. Fabian, P.C. M. Christianen, R. Bratschitsch, C. Schüller, and T. Korn, Nano Lett. 16, 7899 (2016).

[65] M. Koperski, M. R. Molas, A. Arora, K. Nogajewski, M. Bartos, J. Wyzula, D. Vaclavkova, P. Kossacki, and M. Potemski, 2D Mater. 6, 015001 (2018).

[66] A. V. Stier, K. M. McCreary, B. T. Jonker, J. Kono, and Scott A. Crooker, Nat. Commun. 7, 10643 (2016).

[67] P. E. Blöchl, Phys. Rev. B 50, 17953 (1994).

[68] G. Kresse and J. Furthmüller, Phys. Rev. B 54, 11169 (1996).

[69] J. P. Perdew, K. Burke, and M. Ernzerhof, Phys. Rev. Lett. 77, 3865 (1996).

[70] L. Du, M. Liao, J. Tang, Q. Zhang, H. Yu, R. Yang, K. Watanabe, T. Taniguchi, D. Shi, Q. Zhang, and G. Zhang, Phys. Rev. B 97, 235145 (2018).

[71] X.-L. Li, X.-F. Qiao, W.-P. Han, Y. Lu, Q.-H. Tan, X.-L. Liu, and P.-H. Tan, Nanoscale 7, 8135 (2015).

[72] W. Zhao, R. M. Ribeiro, M. Toh, A. Carvalho, C. Kloc, A. Castro Neto, and G. Eda, Nano Lett. 13, 5627 (2013).

[73] K. O’Donnell and X. Chen, Appl. Phys. Lett. 58, 2924 (1991).

[74] G.-B. Liu, W.-Y. Shan, Y. Yao, W. Yao, and D. Xiao, Phys. Rev. B 88, 085433 (2013).

[75] R. Schmidt, A. Arora, G. Plechinger, P. Nagler, A. Granados del Águila, M. V. Ballottin, P. C. M. Christianen, S. Michaelis de Vasconcellos, C. Schüller, T. Korn, and R. Bratschitsch, Phys. Rev. Lett. 117, 077402 (2016). 